\title{
Measuring Video Gambling: Instrument Development and Validation
}

\section{Cathy King Pike}

Objective: This article reports the initial validation of an instrument designed to measure problem gambling on video gambling devices. Methods: Two samples were collected and included:

- individuals at random lists of playing locations for all counties in one state $(n=553)$

- $\quad$ a general public sample also drawn from each county $(n=542)$.

Results: The scales attained high levels of internal consistency reliability and had a stable factor structure. The items predicted with high accuracy pathological and non-pathological gambling, and the scales performed well in examinations of discriminant and convergent construct validity. Conclusions: The research provided strong evidence of internal consistency reliability, as well as content, construct, factorial, and preliminary evidence of known-groups validity.

State legislatures have attempted to offset federal spending cutbacks and decreased revenues with a new "golden gate of opportunity": the legalization of lotteries, casinos, and video gambling devices. By 1993, 37 states had legalized lottery, 4 had video poker machines, and 27 had high-stakes casinos (Volberg, 1996). Rose (1995) predicted that reductions in legalized gambling will be preceded by an extensive proliferation of video gambling devices, dramatic increases in problem gambling levels, and increased under-age gambling. As accessibility to gambling has increased in this country, so has the gross amount of spending on gambling. In 1974, \$17 billion was spent on wagering in the United States compared to \$304 billion and \$330 billion in 1991 and 1992, respectively (Christiansen, 1993; Christiansen \& McQueen, 1992; Kallick, Suits, Dielman, \& Hybels, 1979).

With increased gambling legalization and expenditures, researchers and clinicians have noted an analogous increase in problem gambling among players (Gambino, Fitzgerald, \& Shaffer, 1993; Lesieur \& Blume, 1990; Mobilia, 1993). Although one might think that problem gambling levels would be easy enough to identify, this is not necessarily the case. Problem gambling is often masked by other presenting problems. Researchers have noted the comorbidity of problem gambling with a variety of other addictive behaviors, for example, alcohol and drug problems, sexual addictions, overspending, overeating, and risk-taking behaviors (Gambino et al., 1993; Lesieur, 1988b; Lesieur \& Blume, 1990). Lesieur and Rosenthal (1991) pointed toward a growing understanding among researchers and clinicians of the similarities of problem gambling and other addictive behaviors. In addition, researchers have identified that problem gamblers exhibit a greater incidence of psychiatric symptoms, for example, depression, other affective disorders, and suicidal ideation or attempts (Lesieur \& Blume, 1990; Linden, Pope, \& Jonas, 1986). Problem gambling usually does not occur in a social vacuum. Additional problems 
reported by families of problem gamblers were financial difficulties, work problems or loss of job, relationship problems, psychological and physical abuse, and responses by the spouse to the problem gambler (Lorenz \& Yaffee, 1986).

Lesieur (1988a) reported that he found that $34 \%$ of female problem gamblers $(n=50)$ in their sample had been treated by mental health professionals but had never mentioned gambling to their therapists. Only 4 of these respondents had been referred to Gamblers Anonymous (GA) by their therapists. Given the high incidence of comorbidity and the potential of problem gambling to be masked by other symptoms, researchers in this field have suggested that all psychiatric patients, those with major affective disorders, and those reporting depression be screened for problem gambling, rather than depending solely on client report (American Psychiatric Association, 1994; Lesieur \& Blume, 1990; Lesieur \& Rosenthal, 1991).

Five methods have been used to identify problem gambling: the Diagnostic and Statistical Manual of Mental Disorders (DSM) criteria, questions developed by GA, the Cumulative Clinical Signs Method (CCSM), the Massachusetts Gambling Screen (MAGS), and the South Oaks Gambling Screen (SOGS) (American Psychiatric Association, 1994; Lesieur \& Blume, 1987; Shaffer, LaBrie, Scanlan, \&Cummings, 1994; Volberg\&Banks, 1990). The DSM-IV criteria are used by clinicians to identify pathological gambling. Pathological gambling is diagnosed as a score of 5 or more on the 10 DSM-IV criteria. GA questions were developed to facilitate public awareness of problem gambling behaviors. Both the CCSM and the SOGS are used for clinical diagnosis and in prevalence studies of problem gambling. The MAGS was developed to measure problem gambling among adolescents. All of these methods use dichotomous scoring and focus on general forms of gambling.

Each of the five methods exhibits serious measurement problems. The DSM criteria, GA questions, and the SOGS are all noted for producing false positives (Volberg \& Banks, 1990). No information is available about the reliability and validity of the DSM criteria, the GA questions, or the CCSM as measurement instruments (Lesieur, 1994). Almost all of the SOGS items relate to amounts of money wagered, lost, borrowed, and stolen rather than thoughts, feelings, or behaviors that problem gamblers experience. In addition, the SOGS produced extremely high false positives (50\%) in one study of the general population and in the nontreatment samples of its validation study, even though it has been used extensively in prevalence studies (Stinchfield, 1997). The MAGS, a measure of problem gambling developed for use with adolescents, has only seven items that discriminate effectively for problem gambling (Shaffer et al., 1994).

The above five methods of identifying problem gambling have focused on gambling in general rather than specific forms of gambling. Instruments that focus on general forms of gambling may fail to identify factors that contribute to problem gambling with specific types of gambling activities, for example, (Dickerson, 1993).

Video gambling devices are self-contained computer systems and may contain a number of games, for example, black jack, poker, joker's wild poker, Keno, and bingo. The machines may have flashing lights, sirens, bells, or music to call attention to progressively larger jackpots or to indicate a winning hand. The dollar amount wagered on these machines can range from $\$ 1$ to 
\$100 per hand. Players may also double their bets on whether the next card dealt will be a high or low card. Some machines require a minimum dollar amount of winnings before a player is paid.

Researchers have referred to video gambling devices as the "crack cocaine" of gambling due to their perceived increased risk for pathological gambling and their proliferation (Morgan, Kofoed, Buchkoski, \& Carr, 1996; Rose, 1995). Among the factors that have been studied that are associated with problem gambling on these devices are frequency of play, faster and more rhythmic patterns of play, social learning, irrational cognitive sets, and emotional arousal, for example, anger or depression that facilitates "going on tilt" (Dickerson, 1993). One study of problem gamblers found that video gambling device players lost the most money of those forms of gambling studied in single-occasion play on these devices and met significantly more of the DSM-IV criteria for pathological gambling (Morgan et al., 1996). In fact, nearly 88\% of the sample was found to have pathological video gambling involvement. The finding of Morgan et al. (1996) regarding losses was supported by Rose (1995), who reported dramatic increases in revenues for one state that had three forms of legalized gambling. The state received $90 \%$ of its gambling revenues from video gambling alone during 1993. Dickerson (1993) noted that problem gambling involved a range of psychological, emotional and behavioral, and personality factors. Specifically, problem gamblers use a variety of irrational thoughts, emotions, and behaviors that exacerbate problem gambling. These may include superstitious beliefs and faulty thinking to explain losses, subjective excitement, persistent play, depression, or anger (Dickerson, 1993).

The objectives of this research were to develop an instrument that measures problem video gambling with satisfactory reliability, validity, and classification accuracy in identifying problem video gambling and to identify the extent to which the instrument maintains satisfactory psychometric properties when used to measure a general public sample.

\section{Method}

\section{Samples}

This validation study was conducted via in-person surveys in all 46 counties of one state where video gambling devices were the only form of legalized gambling and where this type of gambling had been legal for only 4 years. Two samples were collected. For one sample $(\mathrm{n}=$ 553), research assistants conducted in-person surveys among individuals at a random list of playing locations. The second sample was a general public sample $(n=542)$ collected from major areas of commerce in each county. Data for both samples were collected through an inperson, self-administered survey approach. Approximately 50 research assistants, most of whom were graduate students, participated in the data collection for this study. Generally, 1 research assistant was assigned to collect data for each county.

To select the playing locations that would be sampled, a random list of state-licensed playing locations was drawn for each county. These locations included casinos, convenience stores, 
restaurants, bars, and other sites in which video gambling devices were located and known to the state's department of revenue.

Data for the general public survey were collected from individuals located in major areas of commerce in each county, for example, main streets in major shopping areas, shopping malls, or other areas of high pedestrian traffic. Research assistants were instructed to go to major areas of commerce in each of the state's 46 counties and collect surveys from the first 10 people who they saw in these areas who appeared to be older than 18 years of age. Although this is a convenience sample, its rigor is strengthened by having included all 46 counties in the state rather than in one or a few locations.

Unless a county had a large number of playing locations, research assistants collected a total of 20 survey responses for each county, that is, 10 each from the playing location and general public samples. For counties with substantially larger numbers of playing locations, research assistants attempted to collect 20 to 30 responses for each of the two samples.

Before beginning data collection, the research assistants participated in training sessions on the methods to be used in the research. They were instructed to sample no more than two persons from each of the randomly sampled playing locations. Research assistants were instructed to approach the first person they saw in a playing location who was not actively engaged in playing a video gambling device. The research assistants were instructed not to interrupt anyone playing a video gambling device. During training, the research assistants reviewed the questionnaires and practiced completing the forms themselves to ensure that they were familiar with the task. These data were not used in any analyses.

\section{Measures}

An instrument containing two scales was developed for this study, using the domain sampling method of instrument construction (Nunnally \& Bernstein, 1994). A variety of resources was used to identify item content:

- an extensive literature review of indicators of problem gambling and on video gambling devices;

- interviews with a clinician about clients' reports of thoughts, feelings, and behaviors related to problem gambling on video gambling devices;

- a review of qualitative responses from an earlier pilot study conducted by a clinician;

- informal discussions with acquaintances having friends or relatives with problem levels of gambling on video gambling devices.

Item identification proceeded by identifying and listing all indicators of problem gambling within the literature and among the other sources listed above. Then, the item content was divided into lists that measured thoughts, emotions, and behaviors related to problem gambling. Because the literature focused on cognitive distortions and the emotional "pull" of problem gambling along with behavioral consequences as important to diagnosis, the item content was divided into two sets: cognitive and emotional indicators and consequences of problem gambling. 
The 45-item Video Gaming Device Inventory (VGDI) was developed and contained two scales: one that measured the cognitive and emotional "pull" of video gambling devices (labeled Interest) and one that measured the effects or consequences of video gambling devices (labeled Effects). (Table 1 provides a list of items for each scale.) All items of the instrument were scored dichotomously. Scale scores are derived by counting each "yes" response as 1 and summing all of these within the scale. Higher scores indicate higher levels of problem gambling. Respondents for this study were instructed that each item describes a thought, behavior, or feeling about playing video gambling devices, and respondents were asked to indicate whether they had ever had these thoughts, behaviors, or feelings by placing a check mark beside "yes" or "no." In addition to the development of the VGDI, a number of variables examined socio-demographic information, gambling frequency and extensiveness, legal and illegal inducements, and other factors related to video gambling devices. Only a portion of these variables will be reported here because not all of them are relevant to the purposes of this validation study.

\section{Results}

\section{Sample Descriptions}

The research assistants varied in the extent to which they kept records of refusal rates in data collection, making estimates of refusal rates less rigorous than desired. When this omission was noted, the research assistants were polled about refusal rates. They reported virtually no or very few refusal rates by potential participants, and this is consistent with what one might hypothesize based on the type of survey (face to face) and the amount of public interest in issues related to video gambling. However, one research assistant reported having been turned away from some playing locations in one county when the business managers began notifying each other of the study. In relation to respondents, the author estimates the response rate to range between $80 \%$ and $90 \%$, which is considered good for face-to-face self-administered surveys (Kalton, 1983).

Respondents from the playing location and general public samples exhibited both some similarities and differences. Table 2 presents information by sample on several sociodemographic and gambling variables. Both samples were similar in ethnic breakdown, length of employment, the percentage who were state residents, and whether they had military experience. The general public sample was somewhat older (about 4 years) than the playing sample, more evenly distributed on gender, somewhat more educated, more frequently married, and more frequently unemployed.

The variables that measured video gambling devices yielded interesting descriptive information related to experience with video gambling devices. As expected, substantially more respondents in playing locations reported that they had played video gambling devices $(n=475$ in the playing location sample compared to $n=216$ in the general public sample). More than twice the percentage in the playing location sample (59.4\%) reported playing from three to four times per month and up to four to five times per week than in the general public sample. However, at the highest category of play (more than six times per week), nearly twice the percentage of respondents in the general public sample than in the playing location sample who had ever played reported that they played at this level. The actual numbers of respondents reporting 
playing video gambling devices at this level were 23 respondents in the general public sample compared to 27 respondents in the playing location sample.

\section{Reliability}

Internal consistency reliability coefficients (coefficient alphas) were calculated for the playing location, general public, and combined samples (Cronbach, 1951). Only those respondents who reported that they had ever played video gambling devices were included in these analyses. Listwise deletions were used in all of these analyses. SEMs were computed for all reliability analyses. Table 3 provides information about the internal consistency reliability analyses that were conducted in this study. No item yielded an "alpha if deleted" that equaled or was higher than the obtained alpha for any of the three samples. Thus, all 45 items as developed were retained in the instrument.

The playing location sample yielded coefficient alphas of .93 and .91 for the scales, whereas the general public sample yielded coefficients of .91 for the Interest Scale and .90 for the Effects Scale. The differences in these alphas are very small, and the computation of the internal consistency reliability seems not to have been substantially affected by the notable difference in the numbers of respondents who had no missing values on the items and who had ever played video gambling devices ( $n=316$ and 318 for each of the scales in the playing location and $n=$ 110 and 115 for each of the scales in the general public samples). Internal consistency reliability analyses (coefficient alphas) then were computed for the combined samples. The scales yielded alphas of .93 and .91. Across all of the samples, the SEMs remained low, ranging from 0.89 to 1.88.

\section{Factor Structure}

Factor analyses were computed for the playing location and the combined samples. The KaiserMeyer-Olkin Measures of Sampling Adequacy for both groupings were very high (.919 and .932, respectively). The Bartlett's Test of Sphericity for each grouping was significant at the .000 level. These analyses indicated that the items within the instrument were sufficiently correlated for conducting factor analyses.

All factor analyses were computed only for those respondents who reported that they had ever played video gambling devices. These analyses used the principal components method with an oblique (direct oblimin) rotation. Two preliminary factor analyses, using principal components, were computed for the playing location sample: one with missing values deleted listwise and another with missing values deleted pairwise. The results did not differ depending on how missing values were handled. Consequently, missing values were deleted listwise for the playing location and combined samples. No factor analysis was attempted on the general public sample alone because there were too few responses of "yes" to some of the instrument items to allow analysis. Table 4 provides the results of the playing location and the combined sample. 
The factor analyses yielded a two-factor solution, as determined by examining the screen plot and percentage of variance accounted for by the factor. This solution emerged as hypothesized with all but two items (44 and 31) loading as expected. The two-factor solution for both analyses explained about $41 \%$ of the variance in the items. In the case of Item 44 , it was hypothesized that this item would measure the effects of video gambling. It, in fact, loaded with almost equal weight on both factors. Item 31was hypothesized to measure the cognitive/emotional pull of gambling. It loaded slightly higher on the scale, as hypothesized, for the playing location sample and equally in the combined sample. Item 44 was retained as an Effects item despite its slightly higher loading on Interest because of theoretical considerations and its performance in the Effects reliability analysis. Item 31 was retained as an Interest Scale item. A few items (23 and 38) had nearly equal loadings on the playing location and combined sample, respectively. The extent to which all other items loaded on a given factor did not differ for either analysis.

In both the playing location and combined samples, the correlations between the two factors were moderate (.455 for the playing location and .449 for the combined sample), indicating that although the two factors shared some variance (about 20\%-21\%), they are measuring components of problem gambling that are distinctly different. Nunnally and Bernstein (1994) recommended combining factors when they are correlated at about .70 or above.

\section{Predictive Ability of Items}

After initial evaluations of the scales' psychometric properties were completed, 23 items were selected from the instrument to measure specific DSMIV criteria for pathological gambling. A similar approach has been used in other studies to examine the ability of instrument items to predict DSM-IV criteria for pathological gambling and develop cutting scores for pathological and transitional (or potential) problem gambling (Shaffer et al., 1994; Stinchfield, 1997). Researchers have used this approach because the DSM-IV criteria contain compound issues for a given criteria and do not lend themselves to measurement as stated.

Table 5 includes the DSM-IV criteria, the items used from the instrument to measure the criteria, and the weights assigned for each item. Of the 23 items, all but 6 were items from the Effects Scale. This is consistent with the DSM-IV criteria, which tend to focus on behavioral indicators or outcomes of pathological gambling. In all but two cases, the total of the items for a criterion score total to 1 . There is no item appropriate from the instrument to measure illegal activities associated with problem gambling (DSM-IV Criterion 8), and the ninth criterion includes more than one problem area. For this criterion, the items to measure problems with jobs or relationships due to gambling were measured separately. Each of the items used to measure the component was weighted, so that if a respondent answered yes to each question of the criterion, the score would equal 1 . This approach maintained the original scoring mechanism of the DSMIV criteria while allowing one to measure discretely each of the two problematic areas for the ninth criterion related to pathological gambling. The DSM-IV criteria allow a total of 10 possible points, with a 5 as indicative of problem gambling. This same scoring mechanism is retained with the items used to measure the DSM-IV criteria. 
After recoding the original scoring of the DSM-IV relevant items, the remaining instrument items were used to predict the extent to which individuals could be classified on DSM-IV relevant items as pathological (scores equal to or greater than 5 ) or non-pathological (scores less than 5). A discriminant analysis was used to accomplish this. The Wilks's Lambda was significant at $.448(\chi 2=183.214, \mathrm{p}=.001)$. About $55 \%$ of the variance in whether someone could be classified as a pathological gambler could be predicted by the remaining items in the scale. All of the items in the instrument used to predict membership as pathological or nonpathological were significant predictors. The Wilks’s Lambdas for each item ranged from .795 to .984. Group membership was accurately predicted for an overall $92.9 \%$ accuracy rate, significantly higher than one might have expected by random probability. Respondents were accurately predicted into the non-pathological category with 95.5\% accuracy and with an accuracy rate of $80.5 \%$ for the pathological gambling category. There were nine false positives and eight false negatives in the sample ( $\mathrm{n}=234$, after listwise deletion).

The Box's M analysis for the discriminant analysis, which is a test of equal population covariance matrices, was significant at the .000 probability level. In prior research, it has been found that when dichotomous items are used in a discriminant analysis, a violation of the population covariance matrices tends to result in reasonably accurate results (Gilbert, 1968; Moore, 1973). To be certain that the above results were not an exception to this general finding, a logistic regression, usually used when assumptions of discriminant analysis are violated, was computed to compare its results with the discriminant analysis. The logistic regression yielded a significant $-2 \log$ likelihood of $66.917(\chi 2=152.915, p=.0001)$. The logistic regression found an overall accuracy rate of $93.36 \%$, with $96 \%$ of respondents correctly predicted in the nonpathological category and $80.49 \%$ correctly predicted as belonging to the pathological gambling category. The logistic regression results were essentially the same as those for the discriminant analysis.

\section{Examinations of Discriminant and Convergent Construct Validity}

Several variables were examined in relation to the VGDI scales to examine discriminant and convergent construct validity. Age, length of employment, and length of time respondents had lived in their current residences were hypothesized to have very low or no relationships with scores on the Interest and Effects Scales, that is, discriminant variables with the VGDI scales. Frequency of play, the greatest amount of money lost in one sitting, the longest period of time playing in one sitting, total amount of money lost playing video gambling devices, and total number of inducements that respondents reported had been provided by gaming houses and that had facilitated longer play were all hypothesized to have low to moderate positive correlations with the Interest and Effects Scales, that is, convergent variables with the VGDI scales. Pearson's correlations were computed to examine these hypotheses. Table 6 provides information about these relationships.

Age, length of employment, and length of time respondents had lived in their current residences had very low correlations with the Interest and Effects Scales. Two relationships were significant at the .05 level, but these variables accounted for less than $1 \%$ of the variance in the Interest and Effects Scales. The remaining variables shared low to moderate correlations with the Interest and 
Effects Scales, with correlations ranging from .143 to .509. All were significant at the .01 level. The amount of variance they accounted for in the Interest and Effects Scales ranged from 2\% to $26 \%$. Of the variables used to examine convergent construct validity, inducements and frequency of play shared the most amount of variance with the Interest and Effects Scales.

\section{Discussion}

As hypothesized, the instrument and scale items performed exceptionally well in these psychometric analyses and provided good support for the content, factorial, and construct validity of the instrument. This instrument can be used in evaluation studies of interventions with problem video gamblers, in prevalence studies of video gambling, and for individual clients with intervention scores compared to baseline scores to analyze changes during treatment.

The internal consistency reliability analyses provided good evidence of the instrument's psychometric soundness. The coefficient alphas for the playing location, general public, and combined samples were all at .90 or above and had small standard errors of measurement (SEMs). Nunnally and Bernstein (1994) wrote that alphas of .90 or above can provide some preliminary evidence of the content validity of a scale. Hudson (1982) wrote that coefficient alphas of .80 and above are sufficient for use with individual clients. In fact, the Interest and Effects Scales performed better in the general public sample than the SOGS or the MAGS have performed in other studies using much larger general public samples (Shaffer et al., 1994; Stinchfield, 1997). A recent study undertaken to examine the psychometric properties of the SOGS in a general public sample yielded a .69 internal consistency reliability coefficient (Stinchfield, 1997).

Two additional results of the internal consistency reliability analyses for the Interest and Effects Scales are worth noting. First, besides maintaining a high level of internal consistency reliability for the playing location sample, the Interest and Effects Scales' reliability coefficients were consistent for a much smaller sample (the general public sample). Second, the internal consistency reliability analyses revealed that every item that had been developed to measure the two scales was an effective indicator of the constructs measured by the scales. The reliability analyses from both the playing location and general public samples provided superb support for the internal consistency of the scales and yielded estimates that were sufficiently high for clinical decision making.

The factor structure remained stable for both the playing location and combined sample, and the factor correlation provided support that the two scales measure two related, but different, components of gambling on video gambling devices. The results of the factor analyses provided good support of the content, factorial, and construct validity of the instrument.

The discriminant analysis and logistic regression were used to determine the extent to which scale items could predict pathological and non-pathological gambling. The ability of items to do this provided strong support of the construct validity of the scales and some preliminary evidence of know-groups validity. In addition, several variables examined the discriminant and convergent construct validity of the Interest and Effects Scales. 
All variables were correlated as hypothesized, and these results indicated very good discriminant and convergent construct validity of the scales.

Further research is needed to examine the concurrent criterion-related validity of the VGDI and its scales. In addition, the instructions provided to respondents need to be evaluated and modified using a time limitation, for example, "the past year." Further research is also needed to determine clinical cutting scores for this instrument. This study would examine further the known-groups type of construct validity, provide further information about the concurrent criterion-related validity, and identify the clinical cutting points for the scales. Identifying the cutting points of the two scales would allow clinicians to make more informed judgments about the extent to which clients are recreational gamblers, in transition (either toward or away from pathological gambling), or pathological gamblers.

In an article that examines government legalization and regulation of gambling, Rose (1995) suggested that government-sanctioned gambling can be expected to continue and that there will be an extensive proliferation of video gambling devices, dramatic increases in problem gambling levels, and increased under-age gambling. As state governments continue to pursue "endless fields of dreams" (p. 15) through legalized gambling, social workers and other helping professionals will need effective tools with which to identify those who gamble solely as a recreational activity, be able to diagnose problem levels of gambling, and provide effective interventions for problem gamblers. This research provides the only instrument known to date that measures gambling on video gambling devices, a type of gambling that has been called by clinicians and researchers the crack cocaine of gambling.

[This space left blank intentionally.] 


\section{List of tables}

\section{TABLE 1: Scale Items for the Video Gaming Device Inventory}

\section{Interest Scale}

1. I have thought that a string of bad luck was a clue that I was close to winning.

2. I have thought that coming very close to winning told me my luck was about to change for the better.

3. I have thought about playing video gaming devices when I was not able to play.

4. I have thought that my level of skill in video gaming devices has played a part in winning.

5. I have thought that certain ways that I have played increased my chances of winning with video gaming devices.

6. I have thought that I could predict when the machine was about to pay out.

7. I have thought about strategies for my next video gaming session.

9. When I have really needed to win, I thought it would happen.

10. I have thought that, if l'd keep playing in a session, l'd ultimately win.

11. I have thought that I have better luck than some people.

12. I have talked often with friends about my winnings from video gaming devices.

13. After a string of bad luck with video gaming devices, I have returned to try to win back my losses.

15. I have spent the last dollar in my pocket while playing video gaming devices.

26. I have been criticized by others for playing video gaming devices.

28. I have concealed from others my losses from playing video gaming devices.

29. I have played for longer periods of time than many others seem to play.

31. Video gaming devices have been a way I celebrated the good things that happened to me.

32. When I felt lucky on a certain day, l've wanted to play more video gaming devices.

33. Video gaming devices have given me a rush of excitement.

36. I have liked the risk involved in playing video gaming devices.

38. I have felt guilty about playing video gaming devices.

41. I have daydreamed about making a big win in video gaming devices.

44. I have felt special when I played video gaming devices.

45. I have liked the feeling of competing for a win against the video gaming devices.

(Continued) 


\section{Table 1 (Continued)}

\section{Effects Scale}

8. I have thought about ways to get money to play video gaming devices.

14. I have tried not to use money on other things that l've set aside for video gaming devices.

16. I have sold things to get money to play video gaming devices.

17. My ambition at work was affected by my playing video gaming devices.

18. I was less efficient at work because of video gaming devices.

19. I have played video gaming devices to get money to pay debts.

20. I have had problems with loved ones because of my playing video gaming devices.

21. I have forgotten to do things for my family or others because of video gaming devices.

22. I have borrowed from friends or family to play video gaming devices.

23. I have tried in the past to stop playing video gaming devices.

24. I have had trouble sleeping because of thinking about playing video gaming devices.

25. I have lost time from work because of video gaming devices.

27. I have concealed from others how much I played video gaming devices.

30. I have felt restless when I couldn't play video gaming devices.

34. I have wished I could start life all over because of video gaming devices.

35. Video gaming devices have helped me forget my troubles.

37. Video gaming devices have helped me feel better when I was "down" about things.

39. I have felt depressed about playing video gaming devices.

40. I have wished I could stop playingtvideo gaming devices.

42. I have been fed up with myself for playing video gaming devices.

43. I have considered suicide at least once because of video gaming devices.

[This space left blank intentionally.] 
TABLE 2: Descriptive Statistics for Playing Location and General Public Samples

\begin{tabular}{|c|c|c|}
\hline Variable & $\begin{array}{l}\text { Playing Location } \\
\quad(\mathrm{n}=553)\end{array}$ & $\begin{array}{c}\text { General Public } \\
\quad(\mathrm{n}=542)\end{array}$ \\
\hline Mean age & 36.57 & 40.21 \\
\hline Median age & 33.00 & 37.00 \\
\hline Standard deviation for age & 13.12 & 15.72 \\
\hline \multicolumn{3}{|l|}{ Gender (\%) } \\
\hline Male & 62.0 & 52.5 \\
\hline Female & 38.0 & 46.8 \\
\hline \multicolumn{3}{|l|}{ Ethnicity (\%) } \\
\hline White & 66.9 & 69.7 \\
\hline Black & 29.4 & 27.2 \\
\hline Other & 3.7 & 3.0 \\
\hline \multicolumn{3}{|l|}{ Educational level (\%) } \\
\hline Grades 1-6 & 4.4 & 2.7 \\
\hline Grades 7-12 & 45.4 & 36.3 \\
\hline Some college/technical school & 35.8 & 37.1 \\
\hline Baccalaureate & 9.6 & 15.3 \\
\hline Some graduate education/degree & 4.8 & 8.6 \\
\hline \multicolumn{3}{|l|}{ Employed (\%) } \\
\hline Yes & 79.5 & 71.3 \\
\hline No & 20.5 & 28.7 \\
\hline \multicolumn{3}{|l|}{ Time in current job } \\
\hline Mean years & 7.34 & 7.77 \\
\hline Median years & 4.0 & 5.0 \\
\hline Range & $0-45$ & $0-50$ \\
\hline \multicolumn{3}{|l|}{ State resident (\%) } \\
\hline Yes & 91.3 & 91.1 \\
\hline No & 8.7 & 8.9 \\
\hline \multicolumn{3}{|l|}{ Marital status (\%) } \\
\hline Single & 38.5 & 33.3 \\
\hline Divorced & 16.5 & 10.9 \\
\hline Married & 39.4 & 53.3 \\
\hline Living with other & 5.5 & 2.5 \\
\hline \multicolumn{3}{|l|}{ Military experience (\%) } \\
\hline Yes & 21.1 & 21.7 \\
\hline No & 78.9 & 78.3 \\
\hline \multicolumn{3}{|l|}{ Ever played video poker (\%) } \\
\hline Yes & 88.6 & 38.8 \\
\hline No & 11.4 & 61.2 \\
\hline \multicolumn{3}{|l|}{ Frequency of play (\%) } \\
\hline Less than 1-2 times per month & 34.7 & 60.8 \\
\hline 3-4 times per month & 26.9 & 14.6 \\
\hline 2-3 times per week & 24.5 & 10.1 \\
\hline 4-5 times per week & 8.0 & 3.0 \\
\hline More than 6 times per week & 5.9 & 11.6 \\
\hline
\end{tabular}


TABLE 3: Reliability Statistics for the Interest and Effects Scales

\begin{tabular}{lccc} 
Scale and Statistics & Playing Location & General Public & Combined \\
\hline Interest & & & \\
Alpha & .93 & .91 & .93 \\
SD & 6.93 & 5.71 & 6.80 \\
SEM & 1.88 & 1.68 & 1.82 \\
Effects & & & \\
Alpha & .91 & .90 & .91 \\
SD & 4.36 & 2.81 & 4.08 \\
SEM & 1.30 & 0.89 & 1.20 \\
\hline
\end{tabular}

[This space left blank intentionally.] 
TABLE 4: Structure Matrices for Playing Location and General Public Samples

\begin{tabular}{|c|c|c|c|c|}
\hline \multirow[b]{2}{*}{ Item } & \multicolumn{2}{|c|}{ Playing Location } & \multicolumn{2}{|c|}{ Combined Sample } \\
\hline & Interest & Effects & Interest & Effects \\
\hline 1. & .66 & .33 & .64 & .33 \\
\hline 2. & .67 & .34 & .64 & .30 \\
\hline 3. & .65 & .53 & .64 & .55 \\
\hline 4. & .58 & .23 & .59 & .22 \\
\hline 5. & .55 & .16 & .59 & .16 \\
\hline 6. & .63 & .22 & .63 & .24 \\
\hline 7. & .44 & .40 & .46 & .40 \\
\hline 8. & .54 & .69 & .53 & .70 \\
\hline 9. & .63 & .41 & .62 & .41 \\
\hline 10. & .72 & .33 & .72 & .33 \\
\hline 11. & .53 & .19 & .55 & .18 \\
\hline 12. & .56 & .24 & .55 & .24 \\
\hline 13. & .66 & .35 & .66 & .38 \\
\hline 14. & .36 & .58 & .35 & .56 \\
\hline 15. & .64 & .41 & .62 & .45 \\
\hline 16. & .39 & .56 & .37 & .59 \\
\hline 17. & .22 & .73 & .18 & .71 \\
\hline 18. & .15 & .61 & .13 & .58 \\
\hline 19. & .52 & .63 & .49 & .67 \\
\hline 20. & .45 & .72 & .42 & .71 \\
\hline 21. & .40 & .70 & .37 & .64 \\
\hline 22. & .50 & .57 & .48 & .60 \\
\hline 23. & .46 & .45 & .44 & .50 \\
\hline 24. & .27 & .63 & .25 & .62 \\
\hline 25. & .21 & .73 & .13 & .66 \\
\hline 26. & .57 & .46 & .57 & .49 \\
\hline 27. & .53 & .59 & .49 & .65 \\
\hline 28. & .66 & .48 & .61 & .56 \\
\hline 29. & .58 & .46 & .56 & .49 \\
\hline 30. & .40 & .72 & .37 & .71 \\
\hline 31. & .56 & .54 & .55 & .55 \\
\hline 32. & .65 & .20 & .67 & .28 \\
\hline 33. & .65 & .31 & .67 & .33 \\
\hline 34. & .17 & .64 & .17 & .63 \\
\hline 35. & .37 & .55 & .41 & .53 \\
\hline 36. & .63 & .21 & .65 & .23 \\
\hline 37. & .47 & .54 & .50 & .59 \\
\hline 38. & .47 & .38 & .45 & .45 \\
\hline 39. & .45 & .55 & .42 & .59 \\
\hline 40. & .40 & .54 & .40 & .60 \\
\hline 41. & .63 & .38 & .64 & .40 \\
\hline 42. & .48 & .55 & .47 & .57 \\
\hline 43. & .11 & .59 & .01 & .57 \\
\hline 44. & .49 & .48 & .49 & .48 \\
\hline 45. & .58 & .17 & .63 & .20 \\
\hline
\end{tabular}

NOTE: Results are rounded to two decimal places. 
TABLE 5: Items Used to Measure DSM-IV Criteria for Pathological Gambling

DSM-IV Criterion and VGDI Item

Score

Assigned

$D S M$ Criterion 1. Is preoccupied with gambling (e.g., preoccupied with reliving past gambling experiences, handicapping or planning the next venture, or thinking of ways to get money with which to gamble)

3. I have thought about playing video gaming devices when I was not able to play.

7. I have thought about strategies for my next video gaming session. $\quad 0.25$

8. I have thought about ways to get money to play video gaming devices. $\quad 0.25$

24. I have had trouble sleeping because of thinking about playing video gaming devices.

$D S M$ Criterion 2. Needs to gamble with increasing amounts of money to achieve the desired excitement

14. I have tried not to use money on other things that l've set aside for video gaming devices.

15. I have spent the last dollar in my pocket while playing video gaming devices.

16. I have sold things to get money to play video gaming devices.

0.25

33. Video gaming devices have given me a rush of excitement.

0.25

$D S M$ Criterion 3. Has repeated unsuccessful efforts to control, cut back, or stop gambling

23. I have tried in the past to stop playing video gaming devices.

0.50

40. I have wished I could stop playing video gaming devices.

0.50

DSM Criterion 4. stop gambling

30. I have felt restless when I couldn't play video gaming devices.

DSM Criterion 5. Gambles as a way of escaping from problems or of relieving a dysphoric mood (e.g., feelings of helplessness, guilt, anxiety, depression)

35. Video gaming devices have helped me forget my troubles.

37. Video gaming devices have helped me feel better when I was "down" about things.

$D S M$ Criterion 6. After losing money gambling, often returns another day to get even ("chasing" one's losses)

13. After a string of bad luck with video gaming devices, I have returned to try to win back my losses.

DSM Criterion 7. Lies to family members, therapist, or others to conceal the extent of involvement with gambling

27. I have concealed from others how much I played video gaming devices. $\quad 0.50$

28. I have concealed from others my losses from playing video gaming devices. 
TABLE 5 Continued

Score

DSM-IV Criterion and VGDI Item

Assigned

DSM Criterion 8. Has committed illegal acts such as forgery, fraud, theft, or embezzlement to finance gambling

No items

DSM Criterion 9. Has jeopardized or lost a significant relationship, job,

or educational or career opportunity because of gambling

Job

17. My ambition at work was affected by my playing video gaming devices. 0.33

18. I was less efficient at work because of video gaming devices. 0.33

25. I have lost time from work because of video gaming devices. 0.33

Significant relationship

20. I have had problems with loved ones because of my playing video gaming devices.

21. I have forgotten to do things for my family or others because of my playing video gaming devices.

0.33

26. I have been criticized by others for playing video gaming devices.

DSM Criterion 10. Relies on others to provide money to relieve a desperate financial situation caused by gambling I

22. I have borrowed from friends or family to play video gaming devices.

NOTE: DSM-IV = Diagnostic and Statistical Manual of Mental Disorders (American Psychiatric Association, 1994); VGDI = Video Gaming Device Inventory. Problem gambling for the $D S M-I V$ is diagnosed as a score of 5 or more of the above DSM criteria.

TABLE 6: Examinations of Discriminant and Convergent Construct Validity

\begin{tabular}{lcc}
\hline Variable & Interest & Effects \\
\hline Age & -.007 & -.015 \\
Length of employment & $.084^{*}$ & -.005 \\
Length of time in current residence & .057 & $.088^{*}$ \\
Frequency of play & .407 & .421 \\
Greatest amount of money lost in one sitting & $.186^{* *}$ & $.230^{* *}$ \\
Longest period of time in one sitting & $.234^{* *}$ & $.291^{* *}$ \\
Total amount of money lost & $.143^{* *}$ & $.197^{* *}$ \\
Inducements provided by gambling establishments & & \\
that facilitated longer play & $.509^{* *}$ & $.399^{* *}$ \\
\hline
\end{tabular}

*Significant at .05 level (one-tailed). * Significant at .01 level (one-tailed). 


\section{References}

1. American Psychiatric Association. (1994). Diagnostic and statistical manual of mental disorders (4th ed.). Washington, DC: Author.

2. Christiansen, E. M. (1993). 1992 gross annual wager: Industry rebounds with 8.4\% handle gain. Gaming and Wagering Business, 14, 12-35.

3. Christiansen, E. M., \& McQueen, P. (1992). 1991 gross annual wager. Gaming and Wagering Business, 13, 22-37.

4. Cronbach, L. J. (1951). Coefficient alpha and the internal structure of tests. Psychometrika, 16, 297-334.

5. Dickerson, M. (1993). Internal and external determinants of persistent gambling: Problems in generalising from one form of gambling to another. Journal of Gambling Studies, 9, 225-245.

6. Gambino, B., Fitzgerald, R., \& Shaffer, H. (1993). Perceived family history of problem gambling and scores on SOGS. Journal of Gambling Studies, 9, 169-184.

7. Gilbert, E. S. (1968). On discrimination using qualitative variables. Journal of the American Statistical Association, 63, 1399-1412.

8. Hudson, W. W. (1982). The clinical measurement package: A field manual. Homewood, IL: Dorsey.

9. $\quad$ Kallick, M., Suits, D., Dielman, T., \& Hybels, J. (1979). A survey of American gambling attitudes and behavior (Research Report Series, Survey Research Center, Institute for Social Research). Ann Arbor: University of Michigan Press.

10. Kalton, G. (1983). Introduction to survey sampling. Newbury Park, CA: Sage.

11. Lesieur, H. R. (1988a). The female pathological gambler. In W. R. Eadington (Ed.), Gambling studies: Proceedings of the 7th International Conference on Gambling and Risk Taking. Reno, NV: University of Nevada.

12. Lesieur, H. R. (1988b). Report on pathological gambling in New Jersey. In, Report and recommendations of the Governor's Advisory Commission on Gambling (pp. 103-165). Trenton, NJ: Governor's Advisory Commission on Gambling.

13. Lesieur, H. R. (1994). Epidemiological surveys of pathological gambling: Critique and suggestions for modification. Journal of Gambling Studies, 10, 385-398.

14. Lesieur, H. R., \& Blume, S. B. (1987). The South Oaks Gambling Screen (SOGS): A new instrument for the identification of pathological gamblers. American Journal of Psychiatry, 144, 1184-1188.

15. Lesieur, H. R., \& Blume, S. B. (1990). Characteristics of pathological gamblers identified among patients on a psychiatric admissions service. Hospital and Community Psychiatry, 41, 1009-1012.

16. Lesieur, H. R., \& Rosenthal, R. J. (1991). Pathological gambling: A review of the literature (prepared for the American Psychiatric Association Task Force on DSM-IV Committee on Disorders of Impulse Control Not Elsewhere Classified). Journal of Gambling Studies, 7, 539.

17. Linden, R. D., Pope, H. G., \& Jonas, J. M. (1986). Pathological gambling and major affective disorder: Preliminary findings. Journal of Clinical Psychiatry, 47, 201-203.

18. Lorenz, V. C., \& Yaffee, R. A. (1986). Pathological gambling: Psychosomatic, emotional and marital difficulties as reported by the gambler. Journal of Gambling Behavior, 2, 40-49. 
19. Mobilia, P. (1993). Gambling as a rational addiction. Journal of Gambling Studies, 9, 121-151.

20. Moore, D. H. (1973). Evaluation of five discrimination procedures for binary variables. Journal of the American Statistical Association, 68, 399.

21. Morgan, T., Kofoed, L., Buchkoski, J., \& Carr, R. D. (1996). Video lottery gambling: Effects on pathological gamblers seeking treatment in South Dakota. Journal of Gambling Studies, 12, 451-460.

22. Nunnally, J. C., \& Bernstein, I. H. (1994). Psychometric theory ( ${ }^{\text {rd }}$ ed.). New York: McGraw-Hill.

23. Rose, I. N. (1995). Gambling and the law: Endless fields of dreams. Journal of Gambling Studies, 15, 15-33.

24. Shaffer, H. J., LaBrie, R., Scanlan, K. M., \& Cummings, T. N. (1994). Pathological gambling among adolescents: Massachusetts Gambling Screen (MAGS). Journal of Gambling Studies, 10, 339-362.

25. Stinchfield, R. (1997, June). Reliability, validity, and classification accuracy of the South Oaks Gambling Screen (SOGS). Paper presented at the 10th International Conference on Gambling and Risk-Taking, Montreal, Canada.

26. Volberg, R. A. (1996). Prevalence studies of problem gambling in the United States. Journal of Gambling Studies, 12, 111-128.

27. Volberg, R. A., \& Banks, S. M. (1990). A review of two measures of pathological gambling in the United States. Journal of Gambling Studies, 6, 153-163. 